AperTO - Archivio Istituzionale Open Access dell'Università di Torino

\title{
Lead-time oriented production control policies in two-machine production lines
}

\section{This is a pre print version of the following article:}

Original Citation:

\section{Availability:}

This version is available http://hdl.handle.net/2318/1543124

since 2016-01-08T17:00:31Z

Publisher:

Elsevier

Published version:

DOI:10.1016/j.ifacol.2015.06.447

Terms of use:

Open Access

Anyone can freely access the full text of works made available as "Open Access". Works made available under a Creative Commons license can be used according to the terms and conditions of said license. Use of all other works requires consent of the right holder (author or publisher) if not exempted from copyright protection by the applicable law. 


\title{
Lead-time oriented production control policies in two-machine production lines
}

\author{
Alessio Angius* Marcello Colledani ${ }^{* *}$ András Horváth* \\ * Dept. Computer Science \\ Università di Torino \\ C.So Svizzera 185, Torino \\ (e-mail: $\{$ angius,horvath\}@di.unito.it). \\ ** Dept. Mechanical Engineering \\ Politecnico di Milano \\ via la Masa 1, Milano \\ (e-mail: marcello.colledani@polimi.it)
}

\begin{abstract}
The ability of meeting the target production lead times is of fundamental importance in modern manufacturing systems producing perishable products, where the product quality or value deteriorates with the time parts spend in the system, and in manufacturing contexts where strict lead time constraints are imposed due to tight shipping schedules. In these settings, traditional manufacturing system engineering methods and token-based production control policies loose effectiveness as they aim at achieving target production rates while minimizing the inventory, without directly taking into account the effect on the lead time distribution. In this paper a production control policy for unreliable manufacturing systems that aims at maximizing the throughput of parts that respect a given lead time constraint is proposed for the first time. The proposed policy jointly considers the actual level of the buffer and the state of the second machine in the system and stops the part loading at the first machine if there is unacceptable risk of exceeding the lead-time constraint. The effectiveness of this new policy against the traditional Kanban policy is quantified by numerical analysis. The results show that this new policy outperforms the Kanban policy by providing a tighter control on the production lead time. This approach paves the way to the introduction of new lead-time oriented production control policies to maximize the effective throughput in real manufacturing systems.
\end{abstract}

Keywords: manufacturing systems, lead time, perishable products, production control policy, multiple failure mode

\section{INTRODUCTION AND OBJECTIVES}

Product quality and delivery reliability are key factors for success in modern manufacturing industries. To achieve desired target performance in both indicators, advanced production planning models have been widely analyzed in the past. Manufacturing System Engineering methods have been developed in the last decades for investigating the dynamic behavior of manufacturing systems, for estimating their performance and for supporting their efficient design, operation and improvement. Traditional system design approaches aim at maximizing the production rate of the system, i.e. the average number of parts produced in a given time. However, by only looking at the production rate maximization, undesired side effects can be generated on the system lead time, also referred as system flow time, waiting time or residence time. In particular, since increasing the buffers increases the average production rate of the system but also increases the average lead time, a serious risk of undermining delivery reliability by excessive lead times is encountered.

Limiting the lead time is of particular interest in production systems where strict lead time constraints are imposed due to tight finished product distribution and shipping schedules (Biller et al., 2013) imposed by the customer and in systems producing perishable products. Perishable, or obsolete, or deteriorating products are allowed to spend a limited amount of time inside the manufacturing system. If the system lead time exceeds certain fixed thresholds, the product has to be considered as a defect and has to be scrapped by the system. For example, the food production, and specifically the production of yogurt, is pervaded by strict requirements on hygiene and delivery precision. The production plants have to quickly respond to the market demands and current order situation. A typical production sequence for yogurt includes mixing/standardizing of milk, pasteurization, fermentation, cooling, addition of fruit additives and packaging. The production planning involves very complex problems due to the maximum allowed storage time before packaging. If the time the product flows in the system exceeds this limit, it has to be scrapped. Another example comes from the production of multi-layer, multi-lumen polymeric micro-tubes for medical vascular catheters. During the phase of preparation of the raw material, in forms of granules, the moisture level is measured and if the value exceeds a fixed limit, the granules are dried to reduce the moisture level before the downstream micro extrusion process. The lead time between the drying 
and extrusion processes should not exceed a certain limit to avoid increase of the moisture level by exposure to the air. Excessive moisture level affects the material viscosity. This results in fluctuations of the material shear rate and instability in the extrusion process, thus affecting the quality of the tube section. Other examples of systems with strict lead time requirements due to tight shipping schedules involve, for example, water bottling systems or manufacturing systems integrated into highly responsive supply chains. In these situations, the production system should be operated at the maximal possible production rate under a fixed constraint on the lead time value.

In the literature, production control policies have been proposed to perform an indirect control on the production lead time, by regulating inventory levels. Problems that have been deeply investigated are the optimal selection of parameters in token-based policies (Liberopoulos, 2013) including base-stock, Kanban, Hedging Point Policy (HPP) and hybrid solutions, and the performance comparison of these policies under different scenarios. Moreover, policies for the real-time control of machine parameters, i.e. the processing rates, to regulate the work in process have been proposed (Ma and Koren, 2004), (Toshniwal et al., 2004). Although these policies allow to reduce the production lead time, no direct control of this performance measure is performed. Recently, raw material release policies have been proposed to maximize the throughput under an average lead time constraint. For example, in (Biller et al., 2013) the average production rate in isolation of the raw material release stage is controlled in Bernoulli serial lines. In these contributions, the analysis is focused only on the average lead time and the distribution of lead time is not taken into consideration.

In this paper we focus for the first time on production control policies for unreliable manufacturing systems that aim at maximizing the throughput of parts that respect a given lead time constraint, namely effective throughput. The polices proposed in this paper are of threshold type and regulate the part loading in order to find the best possible trade-off between the goal of maximizing the system's production rate and the need of respecting the imposed lead time constraint. Two policies are investigated in the paper for maximizing the throughput of parts that respect desired lead time constraints in a two-machine one buffer system. The first is the traditional single stage Kanban policy. In this policy, the part release at the upstream stage is stopped if the downstream buffer reaches a certain threshold (buffer capacity). The second is a completely new state-dependent policy. This policy jointly considers the actual level of the buffer and the state of the second machine in the system and decides whether to load the part at the first machine based on this observable states. The effectiveness of these policies is compared by numerical analysis. The results show that this new policy outperforms the Kanban policy by providing a tighter control on the production lead time. In our knowledge, this is the first time a similar approach is investigated and validated in the literature.

In practice, if these policies are applied to systems producing perishable products, where excessive lead times cause the production of defective parts, the proposed approach aims at finding the right trade-off between product quality and production logistics performance of the system. Recently, the importance of considering the bilateral relations between production system design and product quality has been highlighted. For example, in (Inman et al., 2013) and (Colledani et al., 2014a) the most recent approaches integrating the two areas are framed and the main results in this area are highlighted. As a matter of fact, only few contributions addressed this problem in a context where perishable products are produced. The design of in-process buffers in industries producing perishable or deteriorating components and products has been addressed in (Liberopoulos and Tsarouhas, 2002). A project to determine cost-efficient ways of speeding up the croissant processing lines of Chipita International Inc. is reported. The installation of a properly sized in-process buffer at a specific point of the line led to a reduction in failure impact on product quality and an increase of the system efficiency. In (Liberopoulos et al., 2007) the author focused on the production rate of asynchronous production lines in which machines are subject to failures. If the failure of a machine is long enough, the material under processing in the upstream machines must be scrapped by the system. In (Wang et al., 2010) a transient analysis is proposed to design the size of the buffers needed in dairy filling and packaging lines. In (Subbaiah et al., 2011) an inventory model for perishable products with random perishability and alternating production rate is proposed. As shown in these works, inventory capacity should be designed by using an integrated quality-logistics approach.

The paper is structured as follows. In the next section the basic modeling assumptions are introduced and the main performance measures of interest in this context are outlined. In Section 3 the new production control policy is formulated. It can be easily seen that the single stage Kanban policy is a sub-case of the proposed policy. In Section 4 the effect of the proposed policy on the system state-space structure is investigated. In Section 5 the system lead time distribution is calculated by an exact analytical approach. In Section 6 the derivation of the optimal policy is discussed, while in Section 7 the proposed policies are compared with respect to several performance measures. The conclusions and future research directions are highlighted in Section 8.

\section{SYSTEM MODELING}

\subsection{Modeling Assumption}

The system is composed of two machines, namely $M_{1}$ and $M_{2}$, and a finite capacity buffer. Finite capacity buffers are used for modeling the single stage Kanban control policy. Thus, to modify the capacity of the buffer, $B$, is equivalent to change the number of Kanbans at the production stage. A discrete time system is considered, i.e., time is divided into slots. Both machines have equal and constant processing times. Time is scaled so that the processing cycle of each machine takes one time unit. If operational, machines start their operations at the same time instant. Machine $M_{i}$, with $i=1,2$, is unreliable and characterized by $F_{i}$ failure modes. In particular, machine $M_{i}$ may fail with probability $p_{i, j}$ whenever it is operational and begins to process a part, where $j \in\left\{1, . ., F_{i}\right\}$. Consequently, for each failure mode, times to failure (TTFs) follow a 
geometric distribution with mean $1 / p_{i, j}$. If the machine is down in mode $j$ it gets repaired in a slot with probability $r_{i, j}$. Thus, for each failure mode, times to repair (TTRs) follow a geometric distribution with mean $1 / r_{i, j}$. For each machine, failure modes are mutually exclusive, in the sense that a machine cannot go down in a certain mode without being repaired from a different failure mode. Therefore, each machine is characterized by a set of states $S_{i}$ with dimensionality $L_{i}=F_{i}+1$. We will denote by $U$ the up state and by $D_{i}$ the $i$ th failure mode. When the machine is operational it processes 1 part per time unit while it does not process parts if the machine is down. The dynamics of each machine can be captured in the transition probability matrix $T_{i}$, which is a square matrix of size $L_{i}$. An example of transition probability matrix for a machine with 3 failure modes is provided below.

$$
\mathbf{T}_{\mathbf{1}}=\left|\begin{array}{cccc}
1-p_{1,1}-p_{1,2}-p_{1,3} & p_{1,1} & p_{1,2} & p_{1,3} \\
r_{1,1} & 1-r_{1,1} & 0 & 0 \\
r_{1,2} & 0 & 1-r_{1,2} & 0 \\
r_{1,3} & 0 & 0 & 1-r_{1,3}
\end{array}\right|
$$

The generic state indicator for this system assumes the form $s=\left(b, \alpha_{1}, \alpha_{2}\right)$, where $b$ is the number of parts in the buffer and $\alpha_{i}$ assumes values in the set $S_{i}$. Under these assumptions, in total $(B+1) L_{1} L_{2}$ states exist. We assume that, in each time slot, the state of the machine is determined at the beginning of the time unit and the buffer content is changed accordingly at the end of the time unit. The upstream machine is never starved and the downstream machine is never blocked. Machine $M_{1}$ is blocked if the downstream buffer is full. Similarly, machine $M_{2}$ is starved if there are no parts in the upstream buffer. Operational Dependent Failures are assumed, i.e., a machine cannot make transitions to other states if it is starved $\left(M_{2}\right)$ or blocked $\left(M_{1}\right)$. The Blocking Before Service (BBS) mechanism is assumed. These assumptions further limit the state space size as some states, for example state $(B, U, U)$, are not reachable and result to be transient states.

We assume that the lead time is constrained and the processed parts are allowed to spend a maximum of $N$ time units inside the system. Therefore, if the lead time is larger than $N$, the lead time constraint is violated and the part cannot be delivered in output from the system, thus it is disposed, when it leaves $M_{2}$.

\subsection{Performance Measures}

The performance measures of interest for this kind of systems are summarized in the following:

- The total production rate of the system, $E$, calculated neglecting the lead time constraint.

- The probability that the lead time $L T$ exceeds the threshold $N: D=P(L T>N)$.

- The effective production rate, $E^{E f f}$, only considering parts that respect the lead time constraint. It is given by $E^{E f f}=E \times(1-D)$.

- The defective production rate, $E^{D e f}$, i.e. the throughput of parts that do not respect the lead time constraint. It is given by $E^{D e f}=E \times D$.

- The yield, $Y$, i.e. the fraction of parts respecting the lead time limit. It is given by $Y=E^{E f f} / E$.
- The average inventory in the system, or work in progress, WIP, i.e. the average amount of parts in the system.

- The average lead time in the system.

\section{STRUCTURE OF THE PRODUCTION CONTROL POLICIES}

If the system behaves as described in Section 2.1, a traditional single stage Kanban production policy is implemented. In other words, machine $M_{1}$ is allowed to load a raw part, to process it and to release it in the downstream buffer, whenever there is at least one slot available in the downstream buffer and independently of the state of the second machine. However, in case machine $M_{2}$ is characterized by multiple failure modes with significantly different repair times, the Kanban level shall be set as a compromise between the effect of long and short failures, thus resulting in a non-negligible fraction of parts exceeding the leadtime constraint.

In order to reduce the amount of parts that exceed the target lead time, in this paper a new state-dependent production control, or part release, policy is proposed for machine $M_{1}$. The rationale of the policy is explained in the following. Whenever the machine $M_{1}$ is operational and machine $M_{2}$ is down in failure mode $j=1, . ., F_{2}$ if there are more than $b_{j}$ parts in the buffer the first machine is prevented from loading, processing and releasing the part into the buffer. Therefore, for each failure mode of the second machine $M_{2}$, a specific threshold $b_{j}$ is defined that limits the number of parts that should be deposited in the buffer, under that specific condition.

More formally, the proposed state-dependent production control policy is characterized by two actions:

- Action 0: load the part. If the machine $M_{1}$ is operational, the part is loaded, processed and released in the buffer by $M_{1}$.

- Action 1: stop part loading. If the machine $M_{1}$ is operational, machine $M_{1}$ does not load, process, and release the part in the buffer.

The policy $u$ acts on the loading of parts at the first machine $M_{1}$ only if the second machine $M_{2}$ is failed. It is defined by the vector $\mathbf{b}_{\mathbf{u}}$ of critical buffer levels, or thresholds. In detail, for each $j=1, . ., F_{2}$ :

- If the buffer level $b \leq b_{j}$ and $M_{2}$ is down in mode $j$ : implement action 0 .

- If the buffer level $b>b_{j}$ and $M_{2}$ is down in mode $j$ : implement action 1.

It is worth to notice that the implementation of the proposed policy does not entail additional complexity with respect to the single stage Kanban policy. Indeed, in addition to the level of the downstream buffer, also the state of the second machine is taken into consideration to implement the selected action. Both state variables are observable in the system. It is also worth to highlight that the single stage Kanban policy can be seen as a sub-case of the new state-dependent policy. Indeed, if $b_{j}=B$ for $j=1, . ., F_{2}$ then the state-dependent policy reduces to the Kanban policy. However, the application of the state- 
dependent policy affects the behavior of the system. This phenomenon will be investigated in the next section.

\section{EFFECT OF THE POLICY ON THE SYSTEM}

A system applying the policy introduced in the previous section can be modeled by a Discrete Time Markov Chain (DTMC). Let us first consider the state space of the DTMC. As long as $M_{2}$ is up, the buffer level cannot increase. If $M_{2}$ is in failure mode $j$, with $j \in\left\{1,2, \ldots, F_{2}\right\}$, and we have $b \geq b_{j}+1$, then the buffer level cannot increase because of the control policy. Accordingly, the maximal buffer level that can be reached in the system is $\max _{j=1,2, \ldots, F_{2}}\left(b_{j}+1\right)$. Let us assume hence $B=$ $\max _{j=1,2, \ldots, F_{2}}\left(b_{j}+1\right)$. The number of states is approximately $(B+1) L_{1} L_{2}$ because at the boundaries (where the buffer is empty or full) some states are not reachable. It is not possible for example that the buffer is empty and both machines are down.

The transition probabilities of the DTMC can be easily derived based on the parameters of the two machines and the control policy. For the sake of saving space we do not provide a detailed description but give examples of transition probabilities. Assume that the system is in state (b,U, $\left.D_{1}\right)$, i.e., $M_{1}$ is up and $M_{2}$ is in failure mode 1 . If $b \leq b_{1}$ then the next state is $(b, U, U)$ with probability $(1-$ $\left.\sum_{i=1}^{F_{1}} p_{1, i}\right) r_{2,1}$ because $M_{1}$ remains up, $M_{2}$ gets repaired, and in this case the buffer level remains $b$. If $b>b_{1}$ then $M_{1}$ is blocked due to the control policy and the next state is $\left(b, U, D_{1}\right)$ with probability $1-r_{2,1}\left(M_{2}\right.$ remains in failure mode 1$)$ and it is $(b-1, U, U)$ with probability $r_{2,1}\left(M_{2}\right.$ gets repaired).

The transition probability matrix of the DTMC will be denoted by $P$. We will denote by $P^{\prime}$ the matrix which contains only those transition probabilities of $P$ that correspond to placing a part in the buffer. I.e., $P_{i, j}^{\prime}=P_{i, j}$ if in state $j M_{1}$ is up and in state $i M_{1}$ is not blocked (due to the control policy or because the buffer is full) and otherwise $P_{i, j}=0$.

\section{PERFORMANCE ANALYSIS}

The lead time of a part can be computed following the approach described in Colledani et al. (2014b) for lines with machines of general complexity and in Shi and Gershwin (2012) for lines with up-down machines. In this paper we give a brief overview of this approach. The first step is to compute the steady state probabilities of the DTMC characterized by $P$. This corresponds to solve a set of linear equations and can be done by standard techniques (see, for example, Stewart (2009)). The vector containing the steady state probabilities will be denoted by $\pi$. The production rate of the system can be obtained by $E=\pi P^{\prime} 1$ where 1 is a column vector of ones. The second step is to obtain the distribution of the state of the system when a part is put in the buffer. This can be done by calculating $\pi^{\prime}=\pi P^{\prime} / E$ which is a stochastic vector and characterizes in which state the system is when a randomly chosen part enters the buffer. The third step is to build a DTMC that models how a part, which is already put in the buffer, leaves the system. A state in this DTMC is a couple $\left(b, \alpha_{2}\right)$ where $b$ is the number of parts in the system and $\alpha_{2}$ is the state of $M_{2}\left(M_{1}\right.$ has not to be considered in this DTMC). The transition probabilities can be derived from the parameters of $M_{2}$. For example, consider the state $\left(b, D_{2}\right)$, i.e., there are $b$ parts in the system and $M_{2}$ is in failure mode 2 . Then the next state is $(b-1, U)$ with probability $r_{2,2}$ and it is $\left(b, D_{2}\right)$ with probability $1-r_{2,2}$. Let us denote the resulting probability transition matrix by $P^{\prime \prime}$. Note that the DTMC characterized by $P^{\prime \prime}$ has an absorbing state, $(0, U)$, which signals the moment when the last part is removed from the system. The initial probability vector for this DTMC, denoted by $\pi^{\prime \prime}$, will be chosen in such a way that it reflects the situation when a random part is put in the buffer. Accordingly, $\pi^{\prime \prime}$ can be easily derived from $\pi^{\prime}$ (they are not the same because $\pi^{\prime}$ and $\pi^{\prime \prime}$ do not refer to the same state space). The probability that the lead time exceeds a given threshold $N$, denoted by $D$, is equal to the probability that the DTMC characterized by $\pi^{\prime \prime}$ and $P^{\prime \prime}$ is not in its absorbing state after $N$ time units, i.e., we have to calculate the vector $\pi^{\prime \prime} P^{\prime \prime N}$ and look at the entry which corresponds to state $(0, U)$.

Most performance measures listed in Section 2.2 can be directly calculated based on $E$ and $D$. The WIP of the system can be calculated in the standard way from $\pi$. The mean lead time can be calculated based on $\pi^{\prime \prime}$ and $P^{\prime \prime}$.

\section{POLICY OPTIMIZATION}

In principle, the parameters of the proposed policy $\mathbf{b}_{\mathbf{u}}$ should be optimized with respect to some economic performance indicators of the system, for example the expected long term profit. However, in practice it is very hard to estimate the cost coefficients to a level of confidence that is significant for the analysis. Moreover, the use of a synthetic economic objective function prevents from observing characteristics behavior of the system that can provide insights on the application of the policy. Therefore, in this paper we focus on production logistics related performance indicators within the proposed production control policy optimization problem. In detail, the objective is to find the set of parameters $\mathbf{b}_{\mathbf{u}}$ that maximize the effective throughput of the system, i.e. the production rate of parts that meet the lead time constraint, under a maximal buffer size constraint. Therefore, the production control problem is:

$$
E^{E f f^{*}}=\max _{u}\left[E^{E f f}(u)\right]
$$

subject to

$$
\max _{j}\left(b_{j}\right) \leq B^{*}
$$

In order to solve this optimization problem, an exhaustive search approach is implemented in this paper. More intelligent optimization algorithms will be tested in future works.

\section{NUMERICAL RESULTS}

\subsection{Effect of the Policy on the Lead Time Distribution}

First we illustrate the effect of the proposed policy on the lead time distribution of the system. We consider a system in which: i) the first machine has a single failure mode with parameters $p_{1,1}=0.01$ and $r_{1,1}=0.1$; ii) the second 

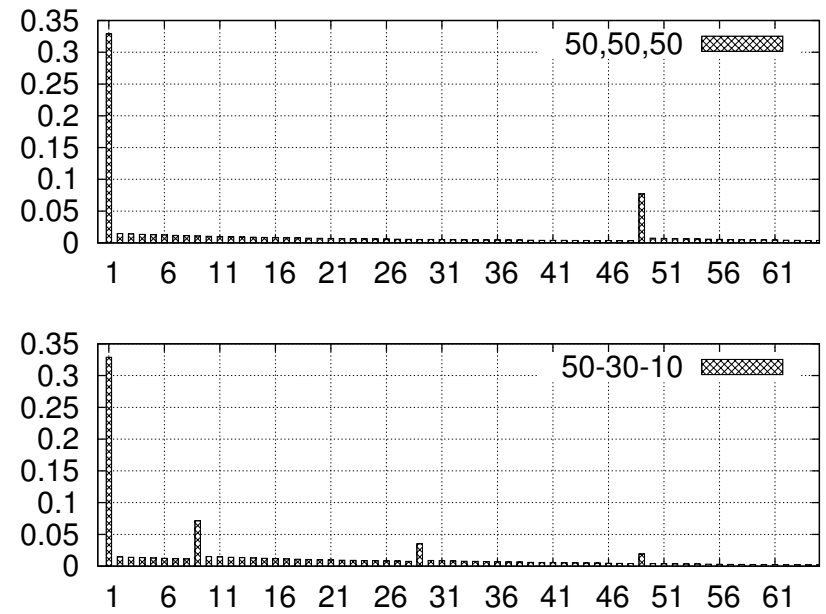

Fig. 1. Lead Time distribution under the Kanban and state-dependent control policies.

machine is characterized by three failure modes that occur with probability $p_{2,1}=0.01, p_{2,2}=p_{2,3}=0.005$, and are repaired with probability $r_{2,1}=0.1, r_{2,2}=0.05$ and $r_{2,3}=0.01$ respectively; iii) the number of Kanbans is fixed at $B=50$ items. As already shown in (Colledani et al., 2014b), under the single stage Kanban control policy (top box of Figure 1) the lead time distribution in a two machine line is characterized by a bi-modal distribution that has peaks at time units 1 and $B-1$. The magnitudes of these peaks depend on the difference of the efficiencies of the two machines.

With the new state-dependent policy, the lead time distribution becomes a multi-modal distribution with as many peaks as the number of failure modes to which a distinct critical threshold level is associated by the control policy. In the second box of Figure 1 we show the lead time distribution of the same system operating under state-dependent control policy with parameters $\mathbf{b}_{\mathbf{u}}=(50,30,10)$. In this case, the lead time distribution is characterized by peaks of different magnitudes at levels $(9,29,49)$. This shift in the distribution can provides considerable benefits to the system behavior. In particular, we can observe that the new peaks detract probability from the tail of the distribution on the right, corresponding to the large values of lead time, thus reducing in practice the probability that the lead time exceeds the fixed constraint. It is remarkable to observe that the introduction of the new policy also reduces the first and the second moments of the distribution, thus providing benefits also to the average lead time of the system. Therefore, these graphs prove that with the introduction of the new state-dependent production control policy a more direct control on the lead time distribution can be achieved. In the next experiment, the impact of the new policy on other system performance measures is investigated.

\subsection{Comparison between policies}

In the second experiment, the performance measures provided by the application of the optimal single stage Kanban and the optimal state-dependent policies are compared for a specific case. We consider a system in which: i)

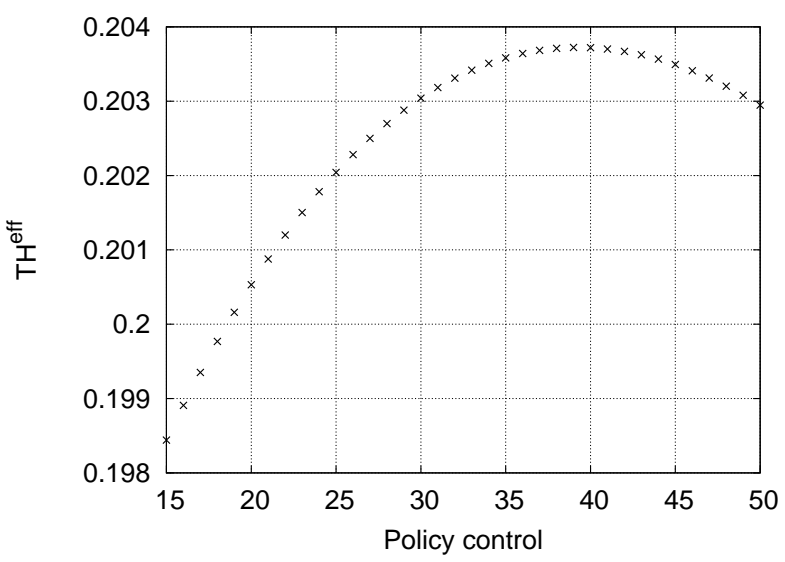

Fig. 2. Effective throughput of the system as a function of the single stage Kanban level.

\begin{tabular}{|c|c|c|c|c|c|c|c|}
\hline $\mathbf{b}$ & $E^{E f f^{*}}$ & $E$ & $E^{D e f}$ & $W I P$ & $\mathbb{E}[L T]$ & $\mathbb{V}[L T]$ & $Y$ \\
\hline Kanban & 0.203 & 0.223 & 0.02 & 16.61 & 74.6 & 21135 & 0.91 \\
\hline New & 0.2041 & 0.218 & 0.014 & 13.47 & 61.68 & 19072 & 0.936 \\
\hline$\Delta \%$ & +0.53 & -2.3 & -30 & -23.3 & -20.5 & -10.8 & +2.8 \\
\hline
\end{tabular}

Table 1. Comparison of the optimal policies for the considered case

the first machine fails with probability $p_{1,1}=0.24$ and is repaired with probability $r_{1,1}=0.01$; ii) the second machine is characterized by three failure modes that occur with probability $p_{2,1}=0.00625, p_{2,2}=0.0125$ and $p_{2,3}=$ 0.003125 , and are repaired with probability $r_{2,1}=0.01$, $r_{2,2}=0.02$ and $r_{2,3}=0.05$ respectively; iii) the lead time constraint is fixed at $N=230$ time units.

We first solve the optimization problem formulated in Section 6 for the single stage Kanban policy. The results are reported in Figure 2. The effective production rate is maximized at $B=39$.

We solved the optimization problem considering the proposed state-dependent policy. Since the second machine is affected by three failure modes there are three threshold levels, i.e. $b_{1}, b_{2}$, and $b_{3}$ to be fixed in order to maximize the effective throughput. The optimal parameters for this case are $\mathbf{b}_{\mathbf{u}} *=(29,39,18)$. The overall set of performance measures is reported in Table 1 for both policies, together with the percentage difference provided by the two policies.

The new state dependent policy provides a slight increase in the effective throughput of the system while the total throughput is decreased. Therefore, under the new production control policy, the system produces less parts in total but more parts respect the lead time constraint. This is due to the fact that the state-dependent policy can prevent the first machine from producing even if it is operational, with the objective to limit the lead time of parts flowing in the system. It is remarkable to highlight the impact on the other performance measures provided by the new policy. With the new policy a higher effective production rate is achieved and at the same time work in progress is reduced by more than $20 \%$. Moreover, the production rate of parts exceeding the lead time constraint is reduced by $30 \%$. This means, in case of perishable products, reducing significantly the fraction of defective parts to be scrapped by the system. Finally, the average lead time is decreased by $20 \%$ and the variance of the lead time is decreased 
by $10 \%$, thus increasing the robustness of the production logistics performance of the system. Similar results have been found over more than 50 cases, not reported here due to space limitations.

\section{CONCLUSIONS AND PROSPECTS}

This paper proposes for the first time the analysis of production control policies for systems with strict lead time limitations. A new state-dependent policy is designed to be implemented in real manufacturing systems where the product is affected by deterioration and perishability and can turn into defective by excessive waiting time in the system. Also, it can be applied in context where the customers requirements on the accepted lead time have to be tightly met. Numerical results prove that the proposed policy can induce a controllable shift in the lead time distribution thus outperforming the application of traditional Kanban policies with respect to the effective production rate. Moreover, it also provides considerable benefits in terms of inventory levels, fraction of parts that exceed the lead time constraint and production yield.

Future research will be oriented towards the extension of this analysis for validating the policy also for production lines with more production stages. Moreover, a formal proof of optimality of the proposed policy within the described context will be investigated. Finally, more complex machine models, also involving non-geometric up and down times and non-identical processing time of machines will be investigated.

\section{REFERENCES}

Biller, S., Meerkov, S., and Yan, C.B. (2013). Raw material release rates to ensure desired production lead time in bernoulli serial lines. International Journal of Production Research, 51, 4349-4364.

Colledani, M., Tolio, T., Fischer, A., Iung, B., Lanza, G., Schmitt, R., and Vancza, J. (2014a). Design and management of manufacturing systems for production quality. CIRP Annals-Manufacturing Technology, 63(2), 773-796.

Colledani, M., Angius, A., and Horvath, A. (2014b). Lead time distribution in unreliable production lines processing perishable products. In 19th IEEE International Conference on Emerging Technologies and Factory Automation (ETFA'14), 1-8.

Inman, R.R., Blumenfeld, D.E., Huang, N., and Li, J. (2013). Survey of recent advances on the interface between production system design and quality. IIE Transactions, 45/6, 557-574.

Liberopoulos, G. (2013). Production release control: Paced, wip-based, or demand-driven? revisiting the push/pull and make-to-order/make-to- stock distinctions. Handbook of Stochastic Models and Analysis of Manufacturing System Operations International Series in Operations Research and Management Science, 192, 211-247.

Liberopoulos, G., Kozanidis, G., and Tsarouhas, P.H. (2007). Performance evaluation of an automatic transfer line with wip scrapping during long failures. Manufacturing and Service Operations, 9/1, 62-83.
Liberopoulos, G. and Tsarouhas, P.H. (2002). Systems analysis speeds up chipita's food processing line. Interfaces, 32/3, 62-76.

Ma, Y. and Koren, Y. (2004). Operation of manufacturing systems with work-in- process inventory and production control. CIRP Annals Manufacturing Technology, $53(1), 361-365$.

Shi, C. and Gershwin, S. (2012). Part waiting time distribution in a two-machine line. In Proc. of the 14th IFAC Symposium on Information Control Problems in Manufacturing, 457-462.

Stewart, W.J. (2009). Probability, Markov chains, queues and simulation: the mathematical basis of performance modeling. Princeton University Press, 1st edition.

Subbaiah, K.V., Rao, S., and Rao, K. (2011). An inventory model for perishable items with alternating rate of production. International Journal of Advanced Operations Management, 3/1, 66-87.

Toshniwal, V., Duffie, N., Jagalski, T., Rekersbrink, H., and Scholz-Reiter, B. (2004). Assessment of fidelity of control-theoretic models of wip regulation in networks of autonomous work systems. CIRP Annals Manufacturing Technology, 60(1), 485-488.

Wang, J., Hu, Y., and Li, J. (2010). Transient analysis to design buffer capacity in dairy filling and packing production lines. Journal of Food Engineering, 98/1, $1-12$. 